\title{
Effect of Polyoxyethylene Alkyl Esters on Permeation Enhancement and Impedance of Skin
}

\author{
Hee-Sun Kim ${ }^{1}$ and Seaung-Youl Oh ${ }^{1, *}$ \\ ${ }^{1}$ College of Pharmacy, Sookmyung Women's University, Seoul 140-742, Republic of Korea
}

\begin{abstract}
In this work, we have investigated the effect of polyoxyethylene alkyl ester nonionic surfactants on percutaneous permeation enhancement of a model drug, ketoprofen. We also investigated the mechanism involved in the enhancement using impedance and solubility measurement. Three groups of nonionic surfactants with different ethylene oxide content were studied. The permeation results showed that all surfactants enhanced the percutaneous absorption, irrespective of the molecular weight. The permeation results from PEG-45 monostearate (PEGMS45) were rather unexpected. Impedance and solubility results indicate that the mechanism involved in the enhancement of permeation by PEG-10 monooleate (PEGMO10) and PEGMS45 is rather different. The results from PEGMS45 suggest that it could be a potential candidate as a skin penetration enhancer with high molecular weight, which may poses less skin irritation and systemic side effect than the smaller surfactant molecules. Overall, this work provided some useful information on percutaneous transport enhancement and the mechanistic insights involved in skin permeation for these nonionic surfactants.
\end{abstract}

Key Words: Polyoxyethylene alkyl ester, Permeation, Impedance, Capacitance, Solubility

\section{INTRODUCTION}

Although the stratum corneum owes its barrier properties largely to its intercellular lipid, it cannot be regarded as a simple lipid membrane due to its special composition and microstructure (Elias et al., 1977). It has been increasingly recognized that understanding the structure and function of the stratum corneum (SC) is essential for achieving effective transdermal drug delivery. For the last 40 or so years, transdermal delivery of drug molecules has been extensively studied. However, only about 20 drug molecules have been formulated successfully as transdermal products, due to the barrier property of SC (Subedi et al., 2010). In order to modify the SC and to increase the permeability, various methods have been developed, including chemical penetration enhancers, prodrugs, iontophoresis, electroporation, sonophoresis and microneedles (Ibrahim et al., 2010; Kigasawa et al., 2010). Among these methods, the simplest way to enhance the skin penetration of drug without any extra device is to incorporate the chemical enhancers into the formulation.

Various hydrophilic and hydrophobic chemicals have been studied for their capability as the skin penetration enhancer and their mechanisms of action have been reported. Lipid extraction and osmotic swelling are reported to be the main reason for the enhancement effect of ethanol (Rastogi et al.,
2001). Lipid barrier of the skin was largely impaired after Isopropyl mirystate treatment (Suh and Jun, 1996). Propylene glycol monolaurate exhibited excellent enhancing capability, and it seemed to affect the local dielectric of the lipid bilayers of the SC without the alteration of lipid packing in SC skin (Cho and Gwak, 2004). For propylene glycol, the mode of action is not clear, though there are some suggestions that it produces some change in the hydration layer in the SC lipid bilayer (Ward and Osborne, 1993). In case of OA, after ATR-FTIR study using deuterated oleic acid (OA), it has been reported that OAs form separate domain in the SC lipid matrix and form permeable defects (Ongpipattanakul et al., 1991). Ultrastructural observations demonstrated both marked disorganization of the intercellular lipid lamellae, as well as the presence of distended lacunae within the stratum corneum in OA/propylene glycol skin (Jiang et al., 2000). Azone is known to change the orderness of SC lipid to a more liquid like structure (Benson et al., 2005). Surfactants are reported to act as solubilizer for lipids in the SC (Trommer and Nuebert, 2006). Though the mechanism of action for these penetration enhancers have been widely studied, the mode of action for penetration enhancement is not conclusive yet, and further explanation needs to be provided for better understanding.

The aim of this work is to investigate the effect of polyoxyethylene alkyl ester nonionic surfactants on permeation

\section{www.biomolther.org}

Open Access DOI: 10.4062/biomolther.2011.19.1.109

pISSN: 1976-9148 elSSN: 2005-4483

Copyright $\odot 2011$ The Korean Society of Applied Pharmacology
Received Oct 4, 2010 Revised Oct 28, 2010 Accepted Oct 28, 2010

\footnotetext{
*Corresponding Author

E-mail: syoh@sookmyung.ac.kr

Tel: +82-2-710-9563, Fax: +82-2-719-6193
} 
enhancement of a model drug, ketoprofen, and to get more mechanistic insights on the permeation enhancement. Three groups of chemically related nonionic surfactants, which are derivatives of lauric acid (LA), stearic acid and OA with different ethylene oxide content, giving differences in hydrophilicity and molecular weight, were studied. The effect on electrical properties of skin was examined for these nonionic surfactants, in order to provide some mechanistic insights into the penetration enhancement through skin. We also have examined the solubility of ketoprofen in various vehicle solutions containing nonionic surfactants and the result was used in combination with the impedance data for the interpretation of permeation results. Skin permeation study for OA and LA were also carried out for comparison.

Nonionic surfactants are widely used in dermal formulations, primarily as stabilizers (Bárány et al., 2000). These are polysorbates (ethoxylated esters, partial esters of sorbitol), polyoxyethylene alkyl ethers, polyoxyethylene alkyl esters, polyoxyethylene alkylphenols, and poloxamers (polyoxyehylene-polyoxypropylene block copolymers). It is thought that these surfactants are mild on the skin even at high loadings and long-term exposure. Their effect on skin permeability has been determined for only a small number of drugs so far (Park et al., 2000) and not much efforts have been made about their direct effects on the lipid of the SC.

Electrically, skin is usually represented as a parallel combination of capacitance $(C)$ and resistance $(R)$ (Yamamoto and Yamamoto, 1976). From the stripping experiments, it has been shown that the SC is mainly responsible for the electrical properties of the skin. The capacitance of the skin is thought to originate from the lipid matrix-keratin cell complex, while the resistance appears to be primarily associated with the current flowing pores (Oh et al., 1993). These pores are mainly locating at appendages on skin, such as hair follicles and sweat glands, though there are some unidentified pathways whose contribution to the flow of current is dependent on the magnitude of current (Cullander and Guy, 1991). Electrical impedance $(Z)$ measurements have shown that $R$ and/or $C$ may be affected by various factors such as hydration, ionic strength of the skin-bathing medium, $\mathrm{pH}$ and chemical treatment (Oh and Guy, 1993). The effect of iontophoresis on $R$ and $C$ has also been studied (Oh and Guy, 1995). It has also been shown that skin permeability is closely related to the electrical resistance of skin (Burnette and Bagniefski, 1988).

\section{MATERIALS AND METHODS}

\section{Materials}

All reagents and chemicals were of the highest commercially obtainable purity. Nonionic surfactants containing ethylene oxide (EO) of various average chain lengths were obtained from Nikko Chemicals Co. (PEG-10 monooleate (PEGMO10), PEG-10 monolaurate (PEGML10), PEG-10 monostearate (PEGMS10), PEG-25 monostearate (PEGMS25), PEG-45 monostearate (PEGMS45)) and Sigma Chemical Co. (PEGmonolaurate 400 (PEGML4), PEG-monooleate 460 (PEGMO4)). OA and LA were purchased from Junsei Chemicals (Tokyo, Japan) and Sigma Aldrich (Seoul, Korea). Chemical structures of these molecules are given in Fig. 1. Sodium phosphate monobasic and HEPES (N-[2-hydroxy-ethyl]-piperasine-n'-12-ethane sulfonic acid) were also purchased from
A<smiles>C[14CH2][14CH2]O[14C](=O)[14CH2][14CH2][14CH2]O</smiles>

B<smiles>CCCC=CCCC(=O)OCCO</smiles>

C<smiles>CCCC(=O)OCCO</smiles>

Fig. 1. Chemical structure of nonionic surfactants (polyethylene glycol alkyl esters). (A) Polyethylene glycol monolaurate (PEGML $m=4,10)$, (B) Polyethylene glycol momooleate (PEGMO $m=4,10)$, (C) Polyethylene glycol monostearate (PEGMS $m=10,25,45)$.

Sigma-Aldrich Co. (Seoul, Korea). Propylene glycol, acetonitrile (HPLC grade) and sodium chloride were obtained from Duksan Chem. (Seoul, Korea). Distilled water was prepared using Barnstead Nanopure ultrapure water system D11921 (Dubuque, lowa, USA). Ketoprofen was provided by Wha-il Chemicals Co. (Ansan, Korea).

\section{Solubility measurement}

Excess amount of ketoprofen was added to the vehicle solution (propylene glycol:water=2:1, v/v), in which the enhancer is dissolved. After equilibration for 24 hours at $37^{\circ} \mathrm{C}$ with shaking, the suspension was centrifuged at $5,000 \mathrm{rpm}$ for $5 \mathrm{~min}$, and the supernatant was diluted with distilled water. The assay of ketoprofen was carried out using a HPLC, as described below.

\section{Skin permeation}

All surfactant molecules (PEGMLs, PEGMOs and PEGMSs) and fatty acids (OA and LA) were investigated for their ability as penetration enhancers. The effect of concentration and molecular weight on flux was studied. Ketoprofen transport experiments were made using full-thickness hairless mouse skin. The skin was excised and frozen immediately after sacrifice of 8-12 week old mouse (Yonsei university lab. Animal center, Seoul, Korea). It was stored at $-70^{\circ} \mathrm{C}$ and then thawed just before use. A side-by-side diffusion cell (Yuil Science, Pusan, Korea) was used. The chamber held a volume of $1 \mathrm{ml}$ and was magnetically stirred. The area of skin exposed to each chamber was $0.5 \mathrm{~cm}^{2}$. The donor and receptor chamber were filled with PG/water (2:1) solution containing ketoprofen (saturated) and HEPES buffer saline $(\mathrm{pH} 7.4)$, respectively. Each enhancer was dissolved in the donor solution. At a predetermined time interval, sampling was made and an equal volume of $1 \mathrm{ml} \mathrm{HEPES}$ buffered saline $(133 \mathrm{mM})$ at $\mathrm{pH} 7.4$ was added to the receptor chamber. Multistirrer Electronicrührer Poly 15 (Variamag, München, Germany) was used for constant stirring of the solutions. Experiments were conducted at $37^{\circ} \mathrm{C}$ using an incubator (Jeio Tech, SI-900, Ansan, Korea). 


\section{Drug assay}

To assay ketoprofen, a high performance liquid chromatograph was employed (Shimadzu LC 10AS). The chromatograph was equipped with an automatic sampler injector (Shimadzu SIL-10A), a variable wavelength UV detector (SPD-10A) and a reversed phase column (KR100-10018, $4.6 \times 250 \mathrm{~mm}$, Kromasil, Bohus, Sweden). The column temperature was maintained at $30^{\circ} \mathrm{C}$. The mobile phase was acetonitrile:phosphate buffer $(\mathrm{pH}=3), 50: 50$. The UV detection was at other $258 \mathrm{~nm}$, with a flow rate $1 \mathrm{ml} / \mathrm{min}$.

\section{Preparation of electrodes}

$\mathrm{Ag} / \mathrm{AgCl}$ electrodes were used for their stability and reversibility. Two types of electrodes were prepared. The rodshaped electrodes were prepared by dipping the tip of an $\mathrm{Ag}$ wire $(99.9 \%$, Aldrich, Milwaukee, WI) (1 mm diameter) into the molten $\mathrm{AgCl}$. The plate-shaped electrodes were prepared electrochemically. Ag plate $(2 \times 2 \mathrm{~cm}, 1 \mathrm{~mm}$ thickness) was lightly sanded with emery paper, washed in a acetone, and then cleaned in $1 \mathrm{M} \mathrm{HCl}$ for $20 \mathrm{~min}$ at $50^{\circ} \mathrm{C}$. After rinsing with distilled water, the $\mathrm{Ag}$ was anodically plated with $\mathrm{AgCl}$ (using a Pt cathode) by immersion in $1 \mathrm{M} \mathrm{KCl}$ and application of a 0.5 $\mathrm{mA}$ current for $24 \mathrm{~h}$.

\section{Diffusion cell}

A side-by-side, two chamber diffusion cell with four electrode inlets (two for signal electrodes and two for sensing electrodes) was designed and made by Yuil Science (Pusan Korea) Inlets in the diffusion cell permitted the positioning of signal electrodes and sensing electrodes on either side of the skin.

\section{Impedance measurements and data analysis}

All solutions for impedance experiments were prepared with distilled water from a nanopure water system (Barnstead).
Skin impedance measurements were made using full-thickness hairless mouse skin, excised and frozen immediately after sacrifice of 8 -week-old mouse. It was stored at $-70^{\circ} \mathrm{C}$ and then thawed just before use. Fig. 2 shows the experimental setup of impedance measurements. The donor and receptor chamber were filled with PG/water (2:1) solution containing enhancer and HEPES buffer saline ( $\mathrm{pH} 7.4)$, respectively. The electric circuit employed for the impedance measurements included a $2 \mathrm{M} \Omega$ resistor in series with the skin. At an applied voltage of $2 \mathrm{~V}$ (peak-to-peak), a sinusoidal current was applied via a signal generator (FG-7002C, LG Precision Co., Seoul, Korea) to the signal electrode on the epidermal side of the skin (the dermal-side electrode was grounded). Since the SC impedance $(Z)$ was routinely $100 \mathrm{~K} \Omega$ or less, the current was determined primarily by the $2 \mathrm{M} \Omega$ resistor in series. The current was therefore can be treated as constant value at all frequencies examined. The potential difference across the skin was then determined by measuring the voltage at each sensing electrode using a digital oscilloscope (OS-902RB, LG Precision Co., Seoul, Korea). The potential difference was evaluated over the frequency $(f)$ range of $1-100 \mathrm{KHz}$, with five frequency points per decade. Each frequency sweep required about 10 min.

Preliminary experiments with skin replacing the model RCcircuit demonstrated that the medium bathing the tissue contributed negligible to the potential drop detected between the sensing electrodes. Data from subsequent experiments using skin were analyzed in the frequency range $(0.1-5.0) \cdot f_{x_{c}} \mathrm{~Hz}$, where $f_{\mathrm{xc}_{\mathrm{c}}}$ is the so-called 'corner' frequency, at which the imaginary component of complex impedance is maximum (as determined in the Cole-Cole plot (36)) and the capacitive effect is most influential. An initial estimate of $f_{\mathrm{xc}}$ was calculated from the equation $f_{x c}=1 / 2 \pi R C$, values of $R$ and $C$ having been derived from the $10 \mathrm{~Hz}$ impedance and the slope of the $1 / Z^{2}$ vs. $f^{2}$ plot. Because the skin cannot be a perfect $\mathrm{RC}$ circuit, and

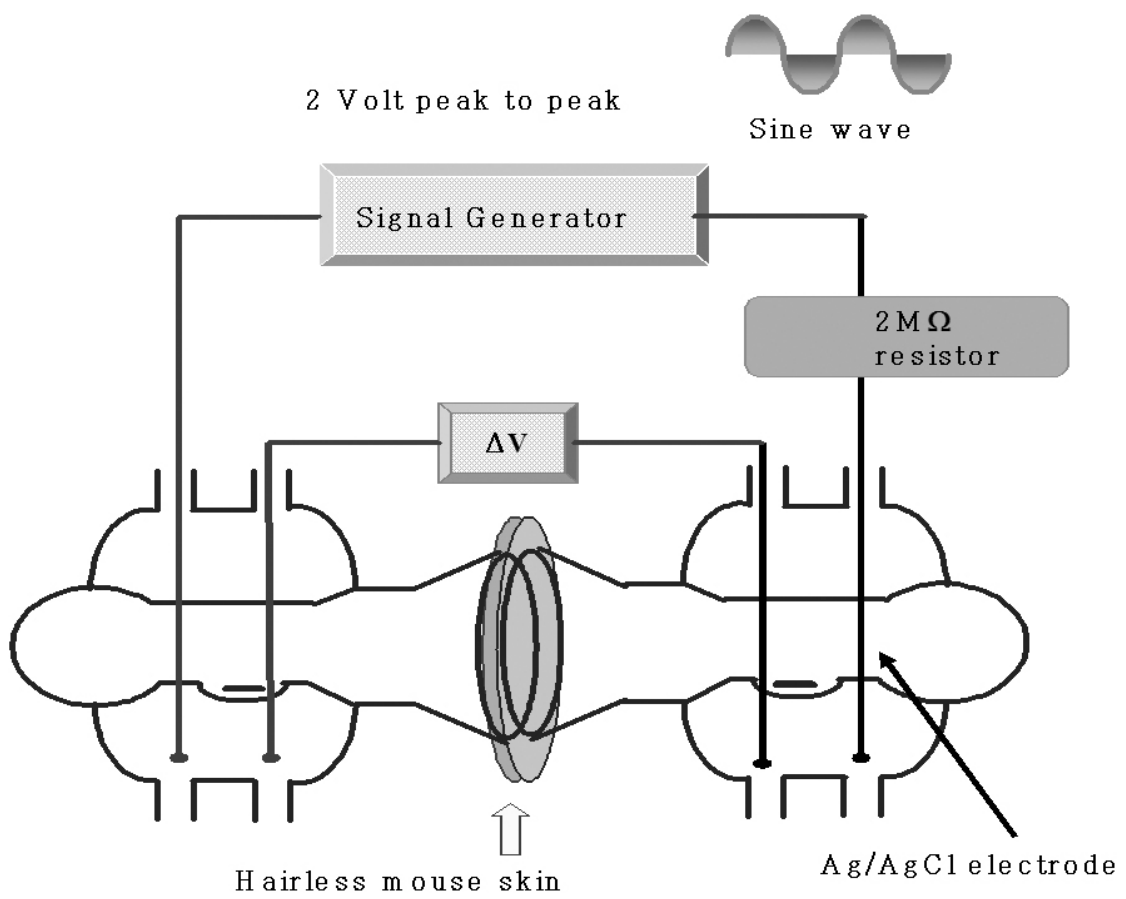

Fig. 2. Schematic diagram of experimental setup for the measurement of impedance. 
shows rather depressed semi-circle, we used low frequency impedance value at $10 \mathrm{~Hz}$ as the $\mathrm{R}$ value, instead of calculating from the intercept of the $1 / Z^{2}$ vs. $f^{2}$ plot. In this way, the frequency range of subsequent $1 / Z^{2}$ vs. $f^{2}$ plots was narrowed, eliminating high frequency data points for which determination of impedance becomes very difficult due to the very small voltages being measured under these conditions.

\section{RESULTS}

\section{Skin permeation}

Nonionic surfactants are commonly used emulsifiers in medicinal and cosmetic formulations. The main components used as nonionic surfactants are the Tween, SPAN and the Brij series. They are known to have enhancing effects on the permeability of biological membranes, including skin (Va Hal et al., 1996). Their comparatively low toxicity and irritation potential make these compounds good candidates as potential penetration enhancers for use in transdermal drug delivery systems. In this work, we have studied 7 kinds of polyethylene glycol alkyl esters for their percutaneous flux enhancement ability and the results are compared. These are PEGML4,
PEGMO4, PEGML10, PEGMO10, PEGMS10, PEGMS25 and PEGMS45 (Fig. 1). Fatty acids such as OA and LA were also studied. Fig. 3 shows the effect of PEGML on the cumulative amount of ketoprofen transported across skin as a function of concentration. Cumulative amount transported for 24 hours $\left(\mathrm{CAT}_{24}\right)$ increased by the application of PEGMLs, and the increase were concentration dependent $(p<0.05)$. When 5 $\%(\mathrm{v} / \mathrm{v})$ of PEGML4 was used, CAT ${ }_{24}$ was $7.9 \mathrm{mg} / \mathrm{cm}^{2}$, which is about 2.6 fold larger than the $\mathrm{CAT}_{24}$ obtained without PEGML4 $\left(3.0 \mathrm{mg} / \mathrm{cm}^{2}\right)$. For $5 \%$ PEGML10, the increase in $\mathrm{CAT}_{24}$ was 2.4 fold, and was smaller than that for PEGML4 $(p<0.05)$. Similar tendency was observed for PEGMO (Fig. 4).

Fig. 5 shows the permeation results of PEGMS10, PEGMS 25 and PEGMS45. When compared to control, $\mathrm{CAT}_{24}$ increased by the application of PEGMSs at all concentration. For $5 \%$ (v/v) PEGMS10, CAT ${ }_{24}$ was $6.1 \mathrm{mg} / \mathrm{cm}^{2}$, and it decreased to $4.7 \mathrm{mg} / \mathrm{cm}^{2}$ when PEGMS25 was used. PEGMS45, which has the longest polyethylene oxide chain, showed similar value $\left(4.7 \mathrm{mg} / \mathrm{cm}^{2}\right)$ to PEGMS25 at $5 \%$. PEGMS45 showed slightly higher $\mathrm{CAT}_{24}\left(5.4 \mathrm{mg} / \mathrm{cm}^{2}\right)$ at $1 \%$ concentration than $5 \%$, though not significant $(p>0.05)$. The comparison for permeation enhancement among these nonionic surfactants is shown in Fig. 6. Both PEGML and PEGMS showed higher
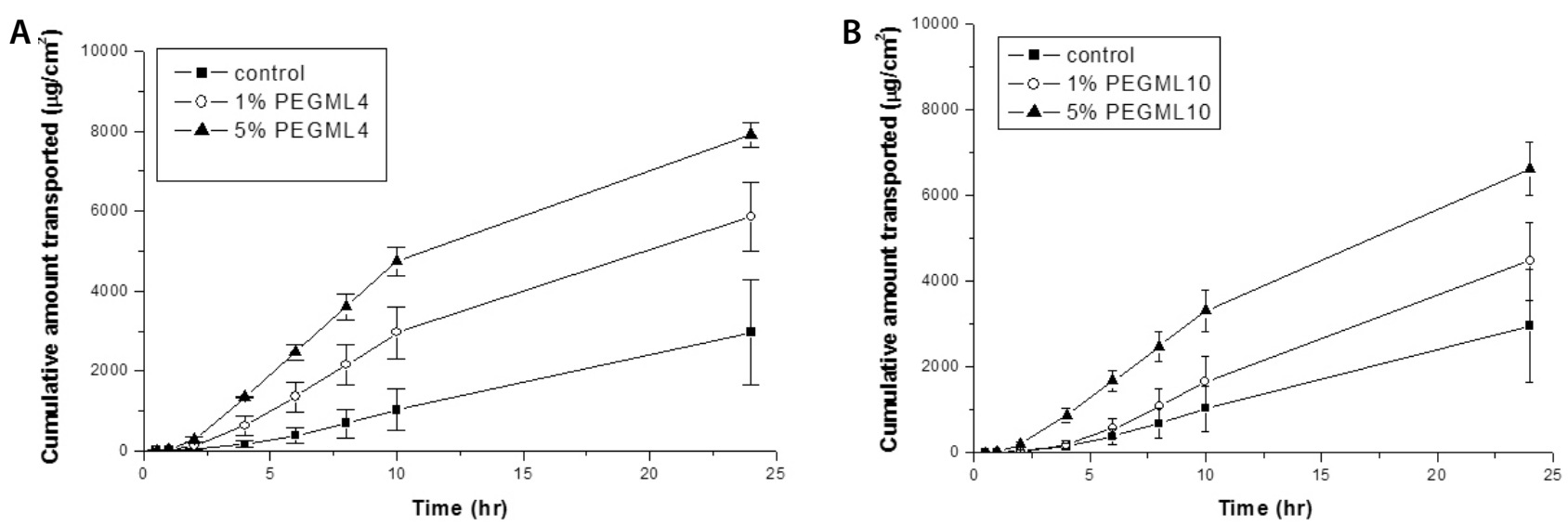

Fig. 3. The effect of PEGML on cumulative amount of ketoprofen transported through hairless mouse skin. (A) PEGML4, (B) PEGML10. Results are expressed as mean \pm S.D. of three experiments.
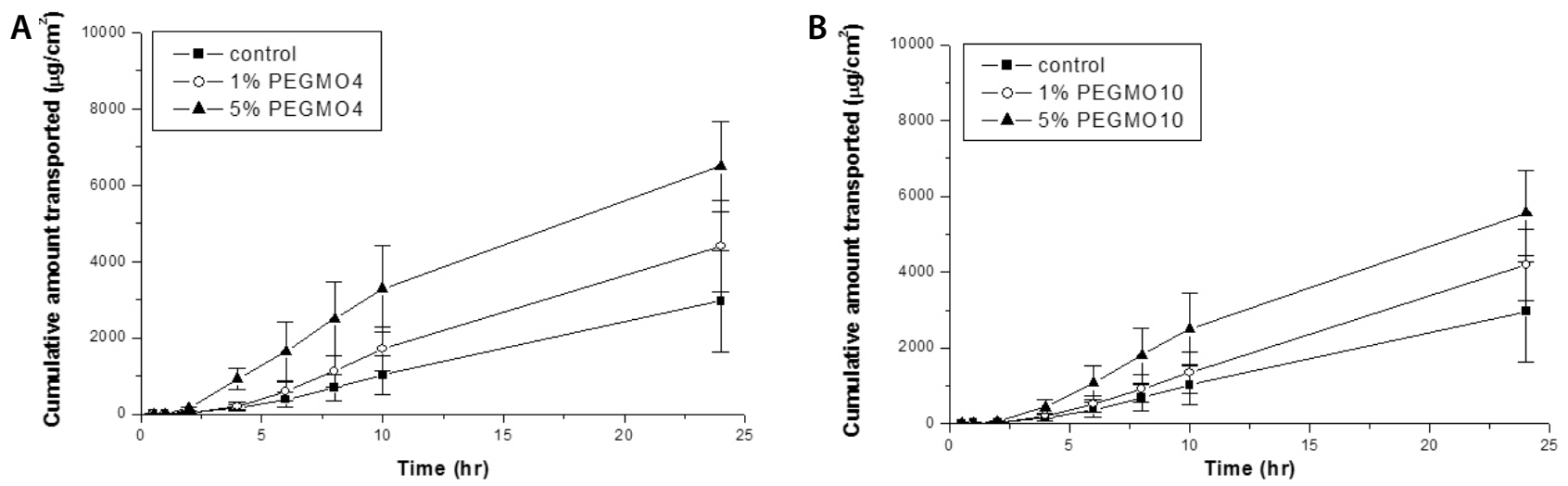

Fig. 4. The effect of PEGMO on cumulative amount of ketoprofen transported through hairless mouse skin. (A) PEGMO4, (B) PEGMO10. Results are expressed as mean \pm S.D. of three experiments. 

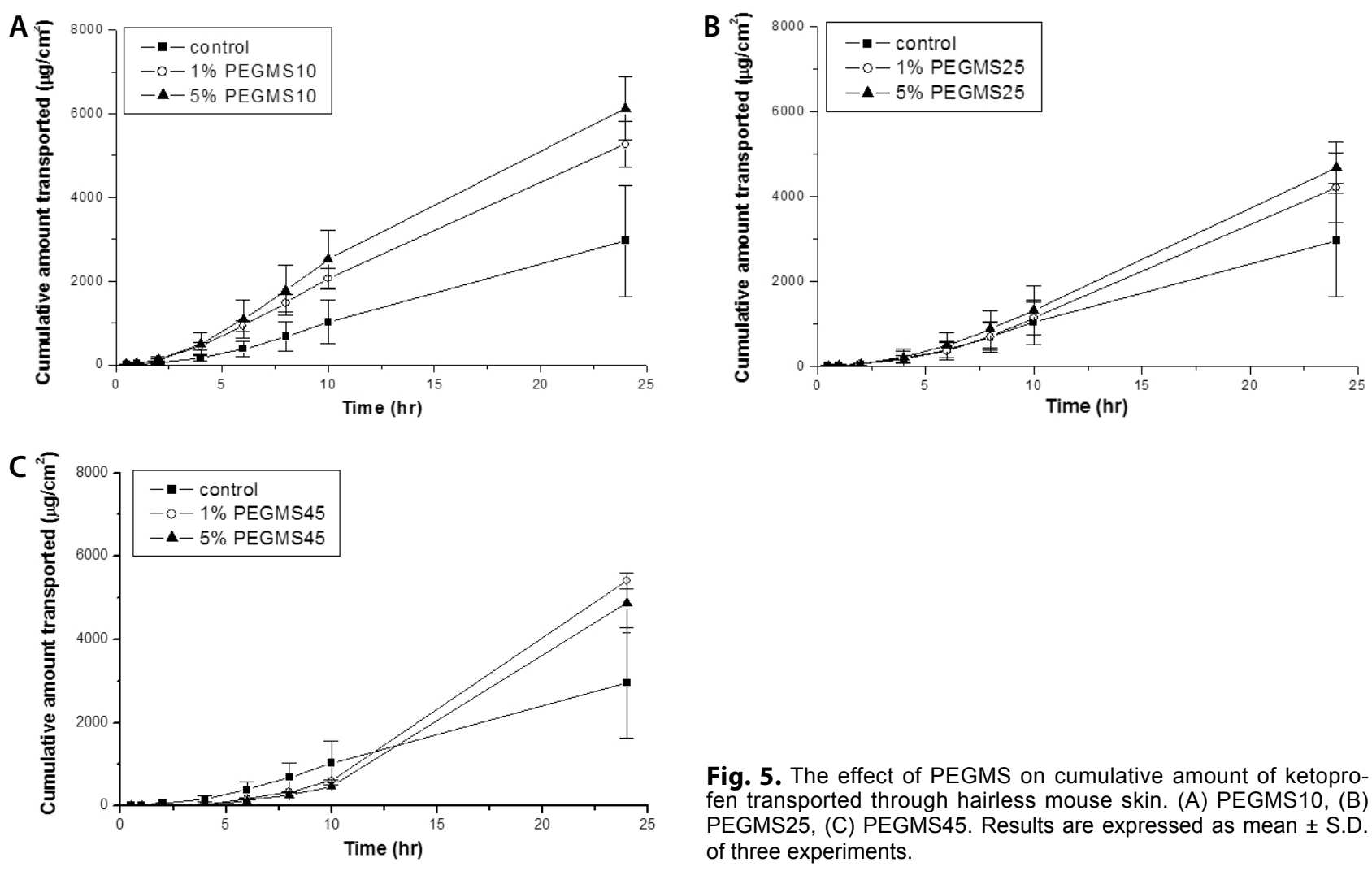

Fig. 5. The effect of PEGMS on cumulative amount of ketoprofen transported through hairless mouse skin. (A) PEGMS10, (B) PEGMS25, (C) PEGMS45. Results are expressed as mean \pm S.D. of three experiments.
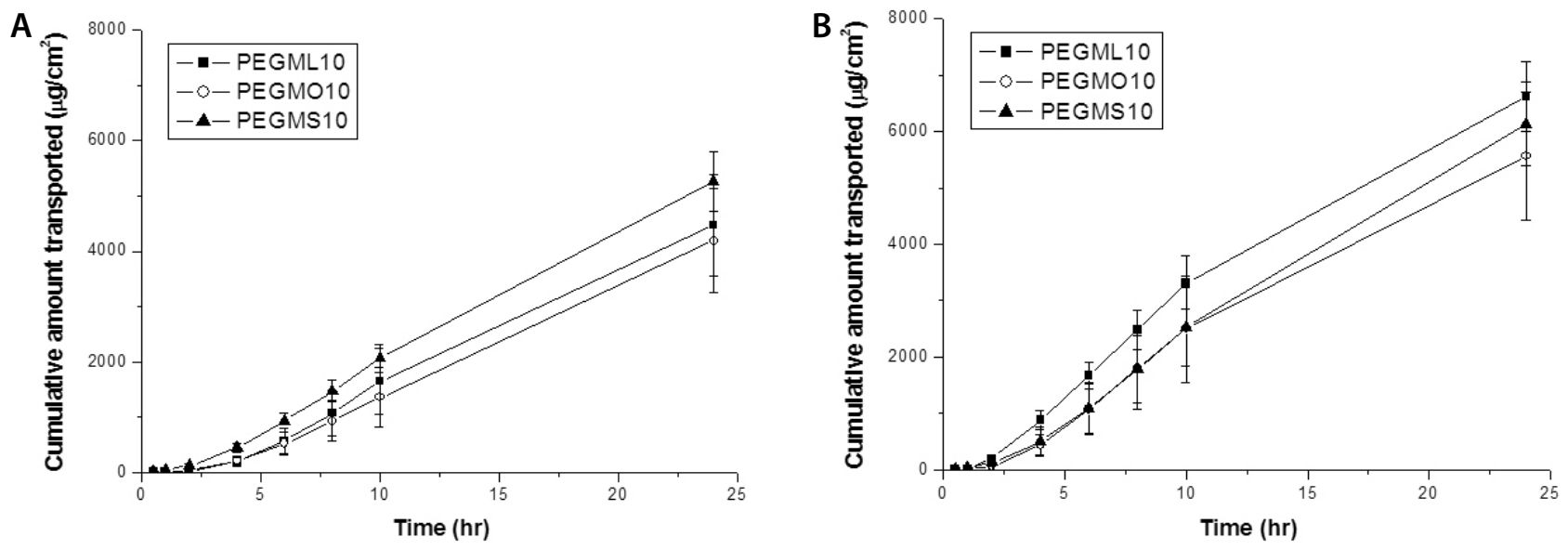

Fig. 6. Flux enhancement effect of PEGML10, PEGMS10 and PEGMO10 through hairless mouse skin at two different surfactant concentration (\% v/v). (A) $1 \%$, (B) $5 \%$.

$\mathrm{CAT}_{24}$ than PEGMO in both concentrations, but it was not significant (one way ANOVA, $p>0.05$ ).

In this work, we also carried out in vitro flux study of lauric and OA without PEG chain for comparison. Fig. 7 shows the permeation profiles of ketoprofen through the skin, these fatty acids are used as penetration enhancers. Stearic acid was not completely dissolved in the donor solution and, hence, penetration experiment could not be carried out. Transport across the skin increased as the concentration of these acids increased $(p<0.05) .5 \%$ LA showed higher enhancing effect than $5 \%$ OA $(p<0.05)$, however, it was not significant at $1 \%$ concentration $(p>0.05)$.

\section{Skin impedance}

Electrically, skin is usually represented as a parallel combination of $C$ and $R$ (Yamamoto and Yamamoto, 1976). The C of the skin is thought to originate from the lipid matrix-keratin cell complex, while the $\mathrm{R}$ appears to be primarily associated with the current conducting pathways (Oh et al., 1993; Burnette and Bagniefski, 1988). It has also been shown that skin permeability is inversely related to the electrical resistance of skin (Burnette and Bagniefski, 1988). In this work, we have inves- 

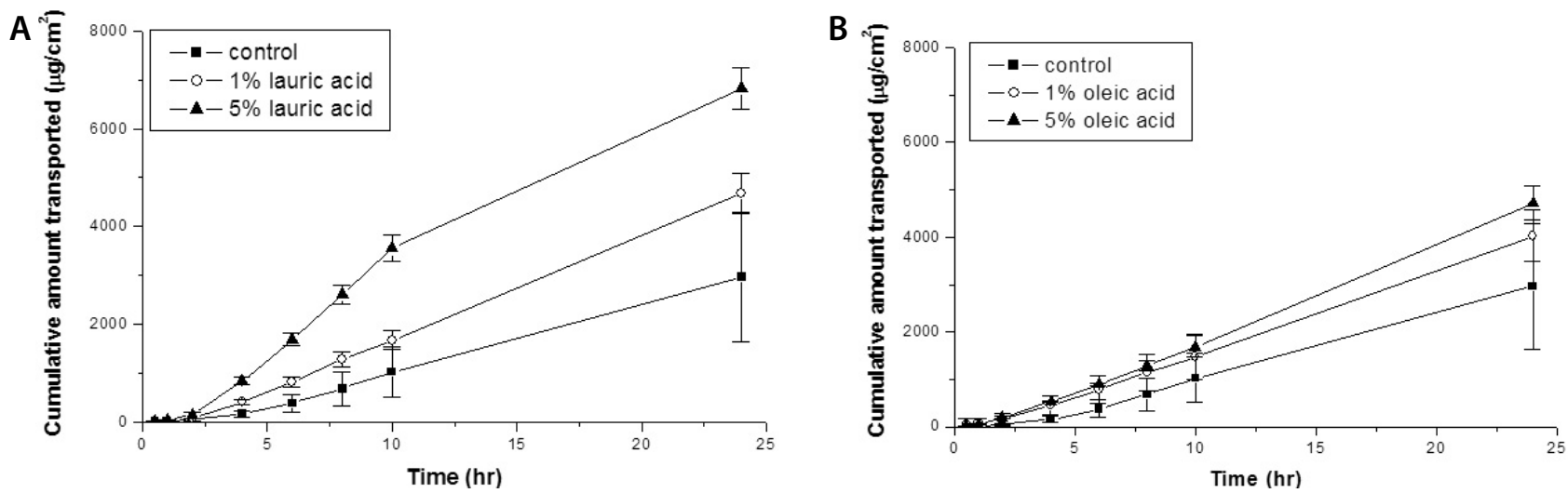

Fig. 7. The effect of lauric acid and oleic acid on cumulative amount of ketoprofen transported through hairless mouse skin. (A) Lauric acid, (B) Oleic acid. Results are expressed as mean \pm S.D. of three experiments.

A

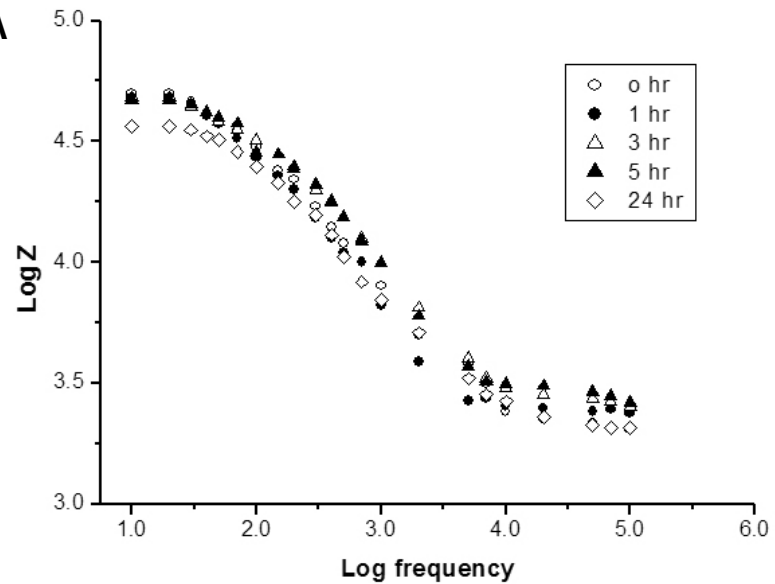

C

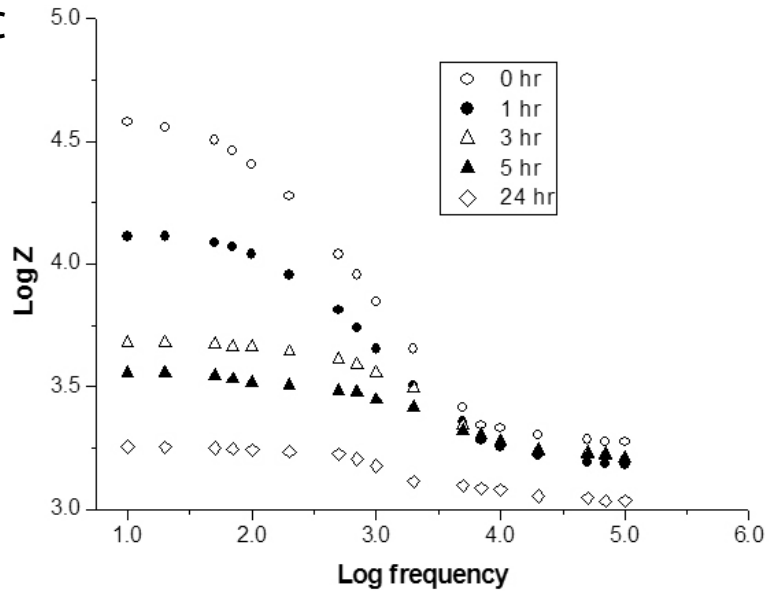

B

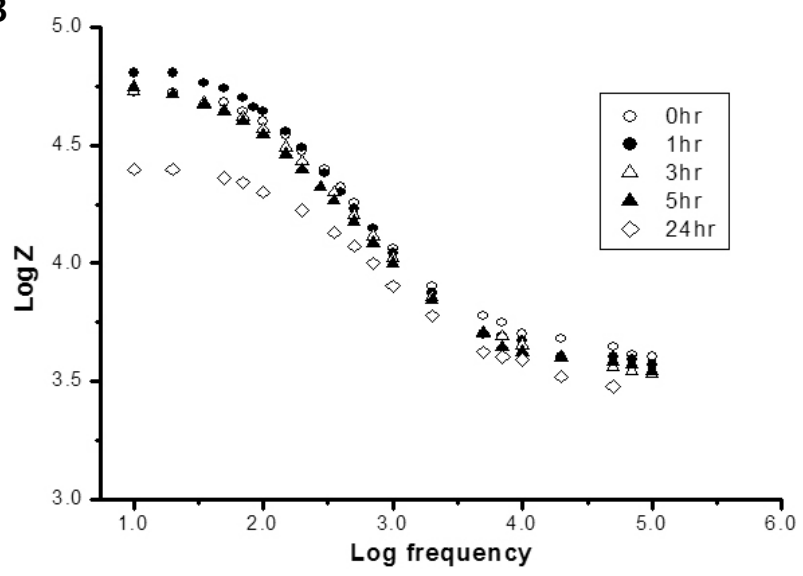

D

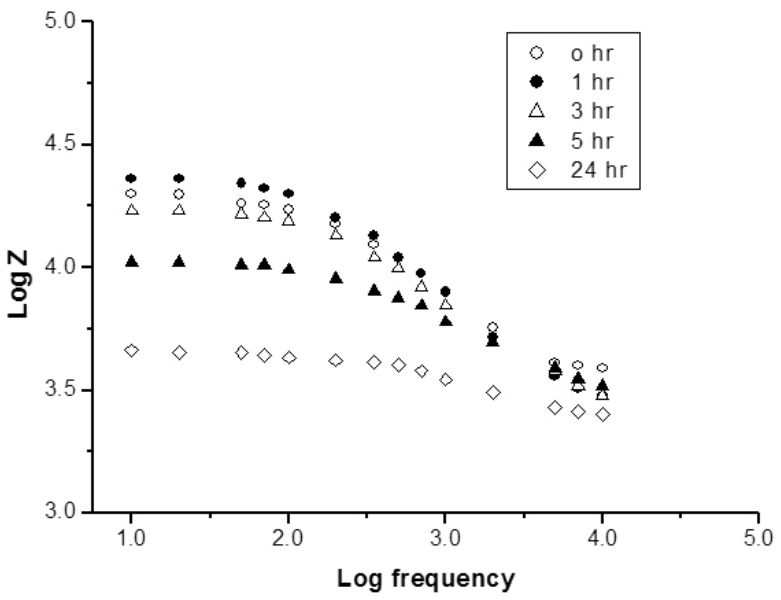

Fig. 8. Bode plot of skin impedance as a function of frequency with time after bathing in donor solution. (A) pH 7.4 buffer, (B) Control solution, (C) Solution containing 3\% (v/v) of PEGMO10, (D) Solution containing 3\% (v/v) of PEGMS45.

tigated the effect of PEG nonionic surfactant on the electrical properties using impedance spectroscopy. $Z$ is measured as a function of frequency and Bode plot is constructed. Electrical resistance and capacitance $(\mathrm{C})$ are calculated and the results are used in the interpretation of the permeation results de- scribed above.

Fig. 8 shows the Bode plot of skin impedance as a function of frequency with time for 24 hours in bathing solutions of control, PEGMO10 and PEGMS45. For comparison, the Bode plot obtained from skin bathing in $\mathrm{pH} 7.4$ buffer is also shown. 
A

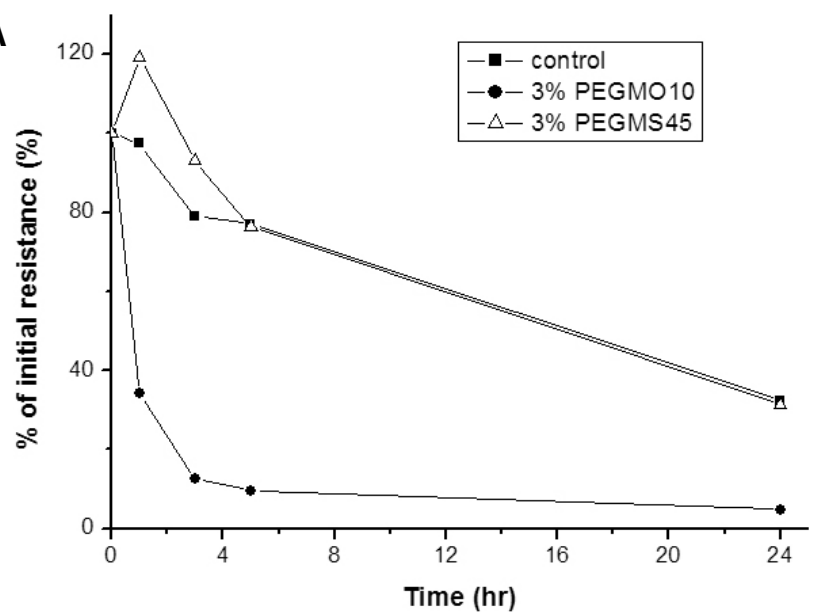

B

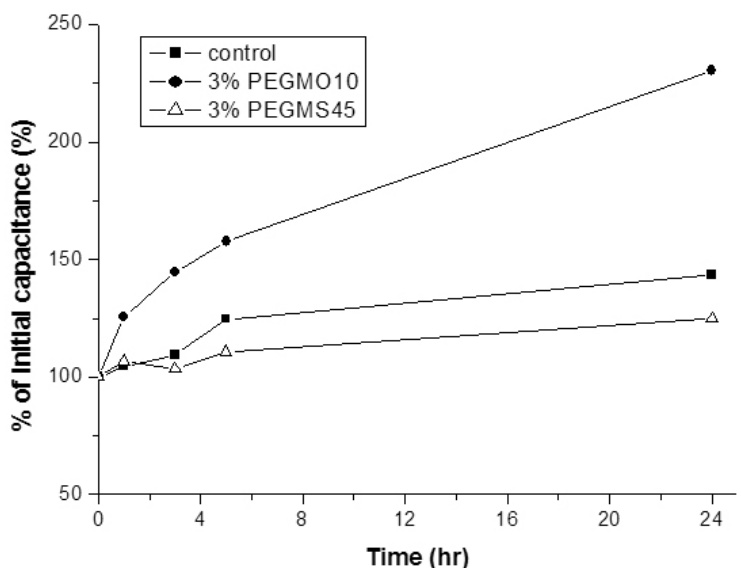

Fig. 9. Change of electrical resistance and capacitance of skin with time after treatment in the bathing solution containing $5 \% \mathrm{v} / \mathrm{v}$ of surfactant. (A) Resistance, (B) Capacitance.

In all cases, the impedance profile with frequency was similar to the typical parallel RC circuit. At low frequency, impedance changed slightly with time, but as the frequency increased, rapid drop in $Z$ occurred due to the conductive role of the capacitor part of the skin. At high frequency, capacitor part of the skin becomes transparent and $Z$ is essentially constant and time-independent. The drop in $Z$ at low frequency with time is small for $\mathrm{pH} 7.4$ buffer solution, however, it was slightly larger for control solution and PEGMS45, and large drop was observed for PEGMO10.

Fig. 9 shows the change in $\mathrm{R}$ and $\mathrm{C}$ of skin with time for PEGMO10 and PEGMS45 treated skin. For control skin, which was immersed in PG/water (2:1) solution, $\mathrm{R}$ decreased slowly with time, and after 24 hours, it reached $32 \%$ of initial $R$ value. PEGMO10 decreased the $R$ rapidly and after 24 hours, $R$ was only $5 \%$ of the initial value. However, for PEGMS45, the decrease in $\mathrm{R}$ with time was much slower than that observed for PEGMO10 and was similar to control skin. Contrary to electrical resistance, $\mathrm{C}$ increased in all cases. For control skin, the increase in $C$ with time was slow, and $C$ reached 144 $\%$ of initial $C$ value. PEGMO10 increased $C$ more rapidly, and it increased more than two fold after 24 hours. However, for PEGMS45, C increased very slowly and it increased only up to $125 \%$ of initial $\mathrm{C}$ after 24 hours.

\section{DISCUSSION}

The permeation results from PEGMS45 were rather unexpected, because we expected that, as the length of the polyethylene oxide chain increases, it would be harder to penetrate into the stratum corneum lipid to increase the flux. Especially, when we think of the molecular weight of PEGMS45, which is about 2,300, it seems difficult for this surfactant to penetrate into the lipid domain. Except for PEGMS25 (Mw: about 1,400 ), the molecular weight of all the other surfactants used are in between 720 and 370 . These molecules seem to be able to partition into the stratum corneum, and perturb the lipid structure.

The permeation results from fatty acid are in good agreement with the results from naloxone permeation or TEWL
Table 1. Solubility of ketoprofen in PG:water (2:1) solution containing different nonionic PEG surfactants in different concentration

\begin{tabular}{lrcc}
\hline & & Conc. $(\mathrm{mg} / \mathrm{ml})$ & Ratio \\
\hline Control & & 82.0 & 1 \\
PEGML4 & $0.1 \%$ & 49.7 & 0.6 \\
& $1 \%$ & 53.4 & 0.7 \\
PEGML10 & $5 \%$ & 66.4 & 0.8 \\
& $0.1 \%$ & 62.2 & 0.8 \\
& $1 \%$ & 73.1 & 0.9 \\
PEGMO4 & $5 \%$ & 82.4 & 1.0 \\
& $0.1 \%$ & 45.6 & 0.6 \\
PEGMO10 & $1 \%$ & 48.6 & 0.6 \\
& $5 \%$ & 65.2 & 0.8 \\
& $0.1 \%$ & 53.1 & 0.6 \\
PEGMS10 & $1 \%$ & 66.5 & 0.8 \\
& $5 \%$ & 73.9 & 0.9 \\
& $0.1 \%$ & 53.6 & 0.7 \\
& $1 \%$ & 58.8 & 0.7 \\
PEGMS25 & $5 \%$ & 87.4 & 1.1 \\
& $0.1 \%$ & 54.9 & 0.7 \\
& $1 \%$ & 65.5 & 0.8 \\
& $5 \%$ & 108.5 & 1.3 \\
& $1 \%$ & 66.4 & 0.8 \\
& $5 \%$ & 111.9 & 1.4 \\
\hline
\end{tabular}

(transepidermal water loss) after different fatty acid treatment. Naloxone skin permeation study showed that permeation through skin increased as the carbon number in saturated fatty acid increases from 7 to 12 (LA) (Aungst et al., 1986). However, it decreased as the carbon number increased further. This parabolic type permeation enhancing effect in fatty acid series was also reported in other studies using testosterone (Yu et al., 1991). Similar results were reported in the study of skin barrier property impairment, using TEWL. TEWL 
increased after treatment with nonanoic acid (9 carbon), and it further increased with undecanoic acid (11 carbon), however it decreased for myristic acid (14 carbon), below the level of control (Kandimalla et al., 1999). The fatty acid results show that low molecular weight PEG ester of lauric or OA (PEGML4 and PEGMO4) exhibited higher permeation enhancing effect than fatty acid itself. For 5\% PEGML4 and PEGMO4 treatment, CAT24 were $7.9 \mathrm{mg} / \mathrm{cm}^{2}$ and $6.5 \mathrm{mg} / \mathrm{cm}^{2}$, respectively. For $5 \%$ LA and OA treatment, CAT24 were $6.8 \mathrm{mg} / \mathrm{cm}^{2}$ and 4.7 $\mathrm{mg} / \mathrm{cm}^{2}$, respectively. Similar result was obtained for $1 \%$ treatment. However, for PEGML10 and PEGMO10, CAT 24 values were similar to those obtained for free fatty acids.

Impedance results indicate that the mechanism involved in the enhancement of permeation by PEGMO10 and PEGMS45 is quite different. DSC and FT-IR study revealed that flux enhancement by LA was due to the increased fluidity of the lipid domain in SC (Aungst, 1989). Increased fluidity of the lipid domain may enhance the hydration of SC, and thus increase the $C$ and decrease the R. For PEGMO10, there was a large change in $R$ and $C$, suggesting that PEGMO10 actually absorbed into the SC and thus disrupted the lipid structure of the inter-corneocyte domain and current conducting pathways. However, for PEGMS45, the change in $R$ and $C$ was minimal and was similar to the values obtained for control experiment, suggesting the possibility that PEGMS45 may not penetrate into the SC. This is highly probable, because the molecular weight of PEGMS45 is about 2300, and PEGMS45 is not expected to diffuse into the SC.

The electrical property data of PEGMS45 make it difficult to provide simple explanation for the skin permeation results. Compared to PG/water solution (control), $\mathrm{CAT}_{24}$ increased nearly two fold after 1\% PEGMS45 treatment. CAT 24 for PEGMS45 was comparable to that of PEGMO10. These results suggest that the ability to enhance skin absorption is similar, despite of the smaller decrease in electrical resistance (Fig. 9), which is known to be inversely related to the skin permeability. One possible speculation is the adsorption of the polymeric enhancer on the SC, and form a layer at the surface to increase the partition coefficient of ketoprofen to the skin surface, which resulted in the enhanced ketoprofen permeation through the skin (Akimoto et al., 2001).

The other point to consider is the change in solubility of ketoprofen in the vehicle by the PEG surfactants. Transdermal permeation of drug molecules can be described by the equation, $\mathrm{J}=\mathrm{DKC} / \mathrm{h}$, where $\mathrm{D}$ is diffusion coefficient, $\mathrm{K}$ is the partition coefficient, $C s$ is the solubility and $h$ is the thickness of the membrane (skin). The increase in permeation can be achieved by the increase in $\mathrm{D}, \mathrm{K}$ and/or $\mathrm{C}_{\mathrm{s}}$. $\mathrm{D}$ can be altered, when the stratum corneum lipid structure between the corneocyte is disrupted by the incorporation of permeation enhancer molecules. $\mathrm{K}$ is determined by the solubility difference between the donor solution and the skin. This means there is a delicate balance between $\mathrm{C}_{\mathrm{s}}$ and $\mathrm{K}$, in their role for the enhancing effect; as the solubility in the vehicle increases, $\mathrm{K}$ decreases. In this work, the solubility of ketoprofen in the donor solution in the absence and presence of the enhancers are carried out, and the results are listed in Table 1, together with the ratio of solubility in donor solution to that in control solution. For small nonionic surfactants with a PEG length less than 10 , the solubility ratio was less than one in nearly all cases, suggesting that permeation enhancing effect of these surfactants is possibly from an increase in $\mathrm{K}$. The increase in
$D$ may also contribute to the permeation enhancing effect due to the disruption of lipid structure by these small molecules, as observed in R \& C change after PEGMO10 treatment (Fig. 9). For larger nonionic surfactants like PEGMS45, the solubility ratio was larger than one at $5 \%$ concentration. Considering the molecular size and the results in Fig. 9 about the change in $\mathrm{R}$ and $\mathrm{C}$ after PEGMS45 treatment, the permeation enhancing effect for PEGMS45 seems not the change in D,

The results from PEGMS45 suggest that it can be a good candidate as a skin penetration enhancer with minimal toxicity, because it may interact with SC minimally and probably not absorbed into the SC lipid domain. Frequent problems with a large number of conventional chemical permeation enhancers are side effects such as skin irritation, allergic reaction and unwanted absorption into the systemic circulation. In literature, various polymeric or oligomeric permeation enhancers have been reported (Aoyagi et al., 1991; Akimoto et al., 2001). Aoyagi et al. synthesized hexadecylpyridinium bromide-type polymers and their permeation enhancing activity on drug penetration was evaluated. Using 5-FU as the model drug for in-vitro permeation study, they showed 2-8 times increase in permeability without any irritation of the skin. They also studied the mechanism using differential scanning calorimetry and concluded that, although the polymeric enhancer interacted with the lipids and proteins of the lipids of the SC, it may not penetrate into deeper layers of the skin. Enhancing ability of polyethylene glycol or polydimethylsiloxane block copolymer with a cationic end-group was also demonstrated (Akimoto et al., 2001). They demonstrated that the chain lengths of both the PEG and PDMS segments are important and suggested that the mechanism of action was an increase in the partition coefficient, rather than a change in the diffusion coefficient through the skin. They also demonstrated the absence of irritation by the application of these polymeric enhancers.

\section{ACKNOWLEDGMENTS}

This Research was supported by the Sookmyung Women's University Research Grants 2008.

\section{REFERENCES}

Akimoto, T., Kawahara, K., Nagase, Y. and Aoyagi, T. (2001) Polymeric transdermal drug penetration enhancer: The enhancing effect of oligodimethylsiloxane containing a glucopyranosyl end group. J. Control. Rel. 77, 49-57.

Aoyagi, T., Terashima, O., Nagase, Y. and Matsui, K. (1991) Preparation of a polymer containing hexadecylpyridinium bromide groups and its utilization as a transdermal drug penetration enhancer. Polym. 32, 2106-2111.

Aungst, B. J. (1989) Structure/effect studies of fatty acid Isomers as skin penetration enhancers and skin irritants. Pharm. Res. 6, 244247.

Aungst, B. J., Rogers, N. J. and Shefter, E. (1986) Enhancement of naloxone penetration through human skin in vitro using fatty acids, fatty alcohols, surfactants, sulfoxides and amides. Int. J. Pharm. 33, 225-234.

Bárány, E., Lindberg, M. and Lodén, M. (2000) Unexpected skin barrier influence from nonionic emulsifiers. Int. J. Pharm. 195, 189-195.

Benson, H. A. (2005). Transdermal drug delivery: penetration enhancement techniques. Curr. Drug Deliv. 2, 23-33.

Burnette, R. R. and Bagniefski, T. M. (1988) Influence of constant current iontophoresis on the impedance and passive $\mathrm{Na}+$ permeability 
of excised nude mouse skin. J. Pharm. Sci. 77, 492-497.

Cho, Y. A. and Gwak, H. S. (2004) Transdermal delivery of ketorolac tromethamine: effects of vehicles and penetration enhancers. Drug Dev. Ind. Pharm. 30, 557-564.

Cullander, C. and Guy, R. H. (1991) Sites of iontophoretic current flow into the skin: identification and characterization with the vibrating probe electrode. J. Invest. Dermatol. 97, 55-64.

Elias, P. M., Goerke, J. and Friend, D. S. (1977) Mammalian epidermal barrier layer lipids: composition and influence on structure. $J$. Invest. Dermatol. 69, 535-546.

Ibrahim, S. A. and Li, S. K. (2010) Chemical enhancer solubility in human stratum corneum lipids and enhancer mechanism of action on stratum corneum lipid domain. Int. J. Pharm. 383, 89-98.

Jiang, S. J., Hwang, S. M., Choi, E. H., Elias, P. M., Ahn, S. K. and Lee, S. H. (2000) Structural and functional effects of oleic acid and iontophoresis on hairless mouse stratum corneum. J. Invest. Dermatol. 114, 64-70.

Kandimalla, K., Kanikkannan, N., Andega, S. and Singh, M. (1999) Effect of fatty acids on the permeation of melatonin across rat and pig skin in-vitro and on the transepidermal water loss in rats in-vivo. J. Pharm. Pharmacol. 51, 783-790.

Kigasawa, K., Kajimoto, K., Hama, S., Saito, A., Kanamura, K. and Kogure, K. (2010) Noninvasive delivery of siRNA into the epidermis by iontophoresis using an atopic dermatitis-like model rat. Int. J. Pharm. 383, 157-160.

Oh, S. Y. and Guy, R. H. (1995) Effects of iontophoresis on the electrical properties of human skin in vivo. Int. J. Pharm. 124, 137-142.

Oh, S. Y., Leung, L., Bommannan, D., Guy, R. H. and Potts, R. O. (1993) Effect of current, ionic strength and temperature on the elec- trical properties of skin. J. Control. Rel. 27, 115-125

Ongpipattanakul, B., Burnette, R. R., Potts, R. O. and Francoeur, M. L. (1991) Evidence that oleic acid exists in a separate phase within stratum corneum lipids. Pharm. Res. 8, 350-354

Park, E. S., Chang, S. Y., Hahn, M. and Chi, S. C. (2000) Enhancing effect of polyoxyethylene alkyl ethers on the skin permeation of ibuprofen. Int. J. Pharm. 209, 109-119.

Rastogi, S. K. and Singh, J. (2001) Lipid extraction and iontophoretic transport of leuprolide acetate through porcine epidermis. Int. J. Pharm. 215, 241-249.

Subedi, R. K., Oh, S. Y., Chun, M. K. and Choi, H. K. (2010) Recent advances in transdermal drug delivery. Arch. Pharm. Res. 33, 339351.

Suh, H. and Jun, H. W. (1996) Effectiveness and mode of action of isopropyl myristate as a permeation enhancer for naproxen through shed snake skin. J. Pharm. Pharmacol. 48, 812-816.

Trommer, H. and Neubert, R. H. (2006) Overcoming the stratum corneum: the modulation of skin penetration. A review. Skin Pharmacol. Physiol. 19, 106-121.

Ward, A. J. I. and Osborne, D. W. (1993) Hydrotropy and penetration enhancement. In Pharmaceutical Skin Penetration Enhancement (K. A. Walters, J. Hadgraft, Eds.), pp. 365-388. Marcel Dekker Inc, New York.

Yamamoto, T. and Yamamoto, Y. (1976) Electrical Properties of the epidermal stratum corneum. Med. Biol. Eng. 14, 151-158.

Yu, J., Chien, T. and Chien, Y. W. (1991) Transdermal Dual-Controlled Delivery of Testosterone and Estradiol: (II) Enhanced Skin Permeability and Membrane-Moderated Delivery. Drug Dev. Ind. Pharm. 17, 1905. 